\title{
Improved Analgesic and Anti-Inflammatory Effect of Diclofenac Sodium by Topical Nanoemulgel: Formulation Development-In Vitro and In Vivo Studies
}

\author{
Shadab Md (1), ${ }^{1,2,3}$ Nabil A. Alhakamy, ${ }^{1,2,3,4}$ Hibah M. Aldawsari, ${ }^{1,3}$ Sabna Kotta, ${ }^{1,3}$ \\ Javed Ahmad, ${ }^{5}$ Sohail Akhter $\mathbb{D}^{6},{ }^{6}$ Md Shoaib Alam, ${ }^{7}$ Mohammad A. Khan, ${ }^{8}$ Zuhier Awan (D), \\ and Ponnurengam Malliappan Sivakumar $\mathbb{D}^{10}$ \\ ${ }^{1}$ Department of Pharmaceutics, Faculty of Pharmacy, King Abdulaziz University, Jeddah, Saudi Arabia \\ ${ }^{2}$ Advanced Drug Delivery Research Group, Faculty of Pharmacy, King Abdulaziz University, Jeddah, Saudi Arabia \\ ${ }^{3}$ Center of Excellence for Drug Research \& Pharmaceutical Industries, King Abdulaziz University, Jeddah, Saudi Arabia \\ ${ }^{4}$ King Fahd Medical Research Center, King Abdulaziz University, Jeddah, Saudi Arabia \\ ${ }^{5}$ Department of Pharmaceutics, College of Pharmacy, Najran University, Najran, Saudi Arabia \\ ${ }^{6}$ New Product Development, Global R\&D, Teva Pharmaceutical Industries Ltd., Runcorn, UK \\ ${ }^{7}$ Research \& Development, Jamjoom Pharmaceuticals, Jeddah 21442, Saudi Arabia \\ ${ }^{8}$ Department of Pharmacology, School of Pharmaceutical Education and Research, Jamia Hamdard, New Delhi 110062, India \\ ${ }^{9}$ Department of Medicine and Biochemistry, Faculty of Medicine, King Abdulaziz University, Jeddah, Saudi Arabia \\ ${ }^{10}$ Center for Molecular Biology, Institute of Research and Development, Duy Tan University, 03 Quang Trung, Da Nang, Vietnam
}

Correspondence should be addressed to Shadab Md; shadabmd1982@gmail.com and Ponnurengam Malliappan Sivakumar; sivamedchem@gmail.com

Received 23 January 2020; Accepted 3 March 2020; Published 21 April 2020

Academic Editor: Muhammad J. Habib

Copyright $\odot 2020$ Shadab Md et al. This is an open access article distributed under the Creative Commons Attribution License, which permits unrestricted use, distribution, and reproduction in any medium, provided the original work is properly cited.

\begin{abstract}
The present study aimed to develop diclofenac sodium nanoemulgel for managing pain and inflammation using the low-energy emulsification technique. Nanoemulsion of diclofenac was formulated using clove oil with adequate amount of surfactants and cosurfactants, and it was converted to hydrogel form using Carbopol 980 as the gelling agent. The droplet size of the oil globules in the nanoemulsion was found to be $64.07 \pm 2.65 \mathrm{~nm}$ with a low polydispersity index $(0.238 \pm 0.02)$ along with high negative zeta potential $(-39.06 \mathrm{mV})$. The developed nanoemulgel exhibited non-Newtonian and pseudoplastic behavior. The in vitro release profile of the developed nanoemulgel was higher as compared to marketed and conventional gel. The carrageenan-induced paw edema test was performed in rats to evaluate the anti-inflammatory activity of developed nanoemulgel. The developed nanoemulgel showed significantly higher $(p<0.01)$ effect in reducing pain and inflammation symptoms as compared to marketed as well as conventional gel of diclofenac. The overall findings of the study suggest that the developed nanoemulgel formulation of diclofenac can be used as a potential approach for the management of pain and inflammation.
\end{abstract}

\section{Introduction}

Nonsteroidal anti-inflammatory drugs (NSAIDs) are the most commonly used drugs to reduce pain and inflammation associated with arthritic disorders [1]. NSAIDs are nonspecific COX (cyclooxygenase) inhibitors; COX is responsible for prostaglandin synthesis, which mediates the process of pain and inflammation [1,2]. Diclofenac sodium (DFS) is globally the most widely prescribed NSAID in management of pain and inflammation in various diseases including rheumatoid arthritis and osteoarthritis [2]. However, long-term use of this drug causes multiple harmful side effects after oral administration such as gastrointestinal bleeding, gastrointestinal ulcers, poor absorption pattern, 
low efficacy, and poor patient compliance [3-5]. Therefore, there is a need for an alternative route for DFS administration to avoid complication related to oral administration.

Topical delivery has been an alternative to conventional systems of various drugs such as analgesics, hormones, scopolamine, and nicotine [6-8]. Topical delivery presents the advantage of avoiding the first-pass hepatic metabolism, thereby improving the bioavailability. However, when exploiting this route, there are some challenges related to drug absorption and permeation. The stratum corneum of skin forms the major barrier for percutaneous penetration of DFS in topical delivery $[9,10]$. Different techniques were used to improve the permeation of different drugs delivered through topical route, such as iontophoresis, microneedle technology, and electroporation, which are not economical. Use of penetration enhancers require higher concentrations, which would result in skin irritancy. Formulations developed for topical delivery are needed to be pleasant to touch and feel or would see a rejection from the patient [10]. Other topical applications of DFS have shown to cause toxicities. A study shows that otic administration of DFS may lead to ototoxicity, which limits its topical usage in chronic otitis [11]. All these challenges show the need for the development of safe and effective topical formulation, which is also economical.

Nanoemulsions have shown to improve the topical delivery of DFS as they show excellent solubilization capacities. Higher oil content in the nanoemulsion system leads to higher penetration of DFS, which was due to the occlusive effect [12]. Systemic delivery of DFS can also be achieved by a combination of nanoemulsion and microneedles; the nanoemulsion improved the bioavailability of DFS [13]. Targeted delivery of DFS has also been achieved by the lipid nanoemulsion system [14]. Nanoemulgel provides various benefits as a topical system as they do not require additional penetration enhancers. The nanoemulsion component has been known to improve the percutaneous permeation, and they also possess dual properties of nanoemulsion and hydrogels. The systems are also known to show a faster rate of drug release compared to creams and ointments. They have a homogeneous behavior and show a hydrogel matrix consistency. A lot of studies were done to improve the topical delivery of DFS, including liposomes to diclosomal gels. Actinic keratosis was locally treated with lecithin vesicles of DFS by local administration [15] which lays the ground for its topical delivery through lipophilic systems. DFS has surfactant like properties, and this was utilized to develop diclosomes (DFS niosomes) which have shown improved permeation compared to traditional gels or even marketed preparation [16]. Topical bioavailability studies of DFS also indicate that it is possible to achieve the IVIVC [17] all of which demonstrates that topical delivery of DFS can be a significant area to pursue research and develop a successful topical system. Given the advantages of the amalgamated system, it was envisioned to improve the percutaneous absorption of DFS and improve the patient compliance through topical route by delivering it through a promising nanoemulgel delivery system. The objective of the present investigation is to formulate, optimize, and characterize nanoemulgel containing DFS formulations for topical delivery. The developed formulation was evaluated for analgesic and anti-inflammatory activity in carrageenaninduced paw edema model.

\section{Materials and Methods}

2.1. Materials. Clove oil, peppermint oil, and eucalyptus oil were purchased from Allin Exporters, Noida, India. Diclofenac sodium (DFS) was obtained from Jamjoom Pharmaceuticals, Jeddah, Kingdom of Saudi Arabia. Isopropyl myristate (IPM), Tween 20, polyethylene glycol 400 (PEG 400), and Carbopol 980 were purchased from Sigma-Aldrich, USA.

2.2. Screening of Formulation Ingredients. The solubility of diclofenac sodium was measured in various oils. An extra amount of diclofenac sodium was added to $2 \mathrm{ml}$ of selected oil in Eppendorf tubes and thoroughly mixed in a vortex mixer, followed by shaking with an isothermal shaker kept at $25 \pm 1^{\circ} \mathrm{C}$ for $72 \mathrm{~h}$. The samples were subjected to centrifugation (Sigma Laborzentrifugen $\mathrm{GmbH}$, Germany) at $3000 \mathrm{rpm}$ for $15 \mathrm{~min}$, and the obtained supernatant was passed through $0.22 \mu \mathrm{m}$ filter syringe. The content of the drug was estimated with UV spectrophotometer at $254 \mathrm{~nm}$ after required dilution with methanol $[18,19]$.

The surfactant was selected for the oil system, which showed the highest drug solubility by determining its emulsification efficiency. The emulsification potential of surfactant was investigated through aqueous titration by drop-to-drop addition of oil phase $(5 \mu \mathrm{l})$ in $20 \%$ aqueous solution of surfactant $(1 \mathrm{ml})$ with the help of a vortex mixer after each addition till mixture of two phases remains clear and transparent [20]. The addition of the oil phase in the surfactant solution phase was continued until turbidity appears. The sample mixture of oil and surfactant phase was kept for $24 \mathrm{~h}$. Then, it is examined visually for any sign of turbidity or phase separation.

The addition of cosurfactants along with surfactant helps to lower the interfacial tension, and it also increases the interface fluidity to ease the formation of nanoemulsion by the low-energy emulsification method. It also helps to obtain nanoemulsion composition with a minimum concentration of surfactant.

2.3. Phase Behavior Study. Phase behavior of the used ingredients, that is, oil phase, aqueous phase, and surfactant mixture, was studied by constructing phase diagram [21]. A phase diagram is a ternary plot, build by performing the aqueous titration and observing the phase behavior after continuous addition of drop-drop water in a mixture of oil and surfactant and cosurfactant (Smix) phase. The sample was vortexed for a few minutes after each addition of aqueous phase and visually observed for the appearance of any turbidity, liquid crystal phase, or phase separation behavior. The proportion of each formulation components was determined after aqueous titration, and ternary plot was created to identify the area of different phase behavior regions. 
2.4. Preparation and Characterization of Nanoemulsion. The nanoemulsion containing diclofenac sodium (DFS-NE) was formulated by the low-energy emulsification method by proper mixing of oil and selected Smix in an optimum ratio with the help of a vortex mixer. The required amount of DFS as per the result of solubility study was dissolved into the optimized oil phase. The oil loaded with the drug was adequately mixed with Smix phase, and purified water was added in small amounts as the continuous phase and immediately mixed by a vortex mixer until a clear, transparent, isotropic system was obtained. The different compositions of DFS-NE were characterized for thermodynamic stability and finally optimized to assess the size distribution and morphology for nanoemulsion droplet.

2.4.1. Thermodynamic Stability Study. The formulated DFS$\mathrm{NE}$ was examined for three heating-cooling cycles at $45^{\circ} \mathrm{C}$ and $4^{\circ} \mathrm{C}$ for $48 \mathrm{~h}$ and monitored for any change in appearance including colour change, drug precipitation, and phase separation [22]. After that, the optimized DFS-NE was centrifuged at a speed of $3500 \mathrm{rpm}$ for 30 minutes to examine phase separation or drug precipitation [22]. Finally, the freeze-thaw cycle was performed six times with alternate freezing and thawing cycles at $-20^{\circ} \mathrm{C}$ and $25^{\circ} \mathrm{C}$ for $24 \mathrm{~h}$ in a deep freezer and room temperature, respectively, to check any change in appearance including phase separation, drug precipitation, and change in colour [22].

2.4.2. Droplet Size and Zeta Potential Analysis. Particle size distribution and zeta potential of the developed nanoemulsion (DFS-NE) were carried out using Malvern Zetasizer Nano-ZS-90 (Worcestershire, UK) [23]. The sample was 100 times diluted with distilled water and mixed uniformly and analyzed at temperature $\left(25^{\circ} \mathrm{C}\right), \lambda \max (633 \mathrm{~nm})$, and at $90^{\circ}$ scattering angle.

2.4.3. Droplet Morphology. The DFS-NE was evaluated for droplet size and morphology analysis by polarized microscope (Nikon Instruments Inc. Melville, NY, US) and transmission electron microscopy (TEM) (JEOL JEM 1010, Tokyo, Japan) after appropriate dilution with distilled water.

2.5. Preparation and Characterization of Nanoemulgel. Diclofenac sodium-loaded nanoemulsion containing nanoemulgel for topical application was formulated using Carbopol 980 as a gelling agent. 0.5\% w/w of Carbopol 980 was first dispersed in purified water under stirring and kept for 1 hour for complete homogeneous swelling. The optimized nanoemulsion formula was homogeneously dispersed in the gel, and $\mathrm{pH}$ was adjusted with triethanolamine to obtain diclofenac sodium-loaded nanoemulgel (DFS-NEG) preparation [24]. The optimized DFS-NEG was evaluated for $\mathrm{pH}$, viscosity, extrudability, spreadability, as well as drug content uniformity.
2.5.1. $p H$. $2.5 \mathrm{~g}$ of DFS-NEG was properly diluted with distilled water $(25 \mathrm{ml})$ [25], and $\mathrm{pH}$ was determined using a digital $\mathrm{pH}$ meter (Mettler Toledo $\mathrm{pH}$ Instruments).

2.5.2. Viscosity. The viscosity of the optimized formulation and placebo gel was determined using rotational viscometer at $25 \pm 0.5^{\circ} \mathrm{C}$ [24].

2.5.3. Spreadability. DFS-NEG spreadability was measured by spreading DFS-NEG on a glass plate having a premarked circle of $2 \mathrm{~cm}$ diameter. After that, another glass plate was placed over the first glass plate and $500 \mathrm{gm}$ of weight was kept on it for $5 \mathrm{~min}$. Finally, the resultant diameter of the circle was measured $[25,26]$.

2.5.4. Extrudability. Extrudability was measured with the help of a lacquered aluminium collapsible tube. The percentage amount of gel extruded from the lacquered aluminium collapsible tube. Weight in grams which is required to extrude a minimum of $0.5 \mathrm{~cm}$ ribbon of gel in 10 seconds was recorded [27]. Extrudability is directly proportional to the quantity extruded.

2.5.5. Drug Content Analysis. The content of DFS in developed NEG was determined by dissolving accurately the weight amount of DFS-NEG (1 gm) of sample in specified amount of methanol solution $(70 \% \mathrm{v} / \mathrm{v})$. The drug extraction was carried out by vortexing the sample for $10 \mathrm{~min}$. The vortex sample was centrifuged at $3000 \mathrm{rpm}$ for $15 \mathrm{~min}$, and supernatant was filtered. The filtered samples were diluted with mobile phase consisting of phosphate buffer (30): methanol (70), and analyzed by RP-HPLC (Shimadzu, Tokyo, Japan) with a UV detector at $254 \mathrm{~nm}$ at a retention time of $5.8 \mathrm{~min}$.

2.6. In Vitro Drug Release Study. An automated six Franz diffusion cell sampling system (SFDC6, LOGAN Inst, NJ, USA) with an effective diffusion area of $1.79 \mathrm{~cm}^{2}$ was used to perform in vitro drug release of DFS using $0.22 \mu \mathrm{m}$ polyvinylidene fluoride (PVDF) membranes [28]. The membrane was soaked in phosphate buffer ( $\mathrm{pH}$ 7.4) and placed between the receptor and donor compartments. The receptor compartments of $12 \mathrm{ml}$ capacity were filled with phosphate buffer ( $\mathrm{pH}$ 7.4) and magnetically stirred $\left(600 \mathrm{rpm}\right.$ ) at $32.0 \pm 0.5^{\circ} \mathrm{C} .0 .4 \mathrm{~g}$ of different samples (DFS-gel, marketed emulgel of DFS, and DFS-NEG) was accurately weighed and added to the donor compartment, and $100 \mu \mathrm{l}$ of aliquots was withdrawn at a fixed time interval $(0.5,1,2,4,6$ and $8 \mathrm{~h}$ ) through the sampling port. DFS gel was prepared by dissolving DFS $(1 \% \mathrm{w} / \mathrm{w})$ in required quantity of propylene glycol and dispersed into $0.5 \% \mathrm{w} / \mathrm{w}$ Carbopol 980 hydrogel to obtain DFS gel of strength $1 \% \mathrm{w} / \mathrm{w}$. The methanol was used along with the receptor medium to maintain the sink condition, and equal volume of fresh receptor solution was replaced immediately after the sampling. The samples were analyzed for the cumulative amount of drug released from a 
different type of formulation at each point by RP-HPLC with a UV detector at $254 \mathrm{~nm}$.

\subsection{In Vivo Study}

2.7.1. Animals. The albino Wistar rats of $200-220 \mathrm{~g}$ weight were used to evaluate the analgesic and anti-inflammatory activity of developed DFS-NEG formulation in comparison to DFS gel and marketed emulgel formulation of DFS. The animals were kept in propylene cages having 4 animals per cage with access to food and water ad libitum. The animals were maintained under standard laboratory conditions of temperature $25^{\circ}+2^{\circ} \mathrm{C}$ and $55 \pm 5 \% \mathrm{RH}$ relative humidity with a $12 \mathrm{~h}$ light-dark cycle. Protocol for the test was approved by Research Ethics Committee (reference no: PH- 108-41), Faculty of Pharmacy, King Abdulaziz University, Jeddah, KSA.

2.7.2. Analgesic Activity. The method described by Khan et al. was adopted for the study [29]. In brief, animals were grouped into four groups, with four rats in each group. Group 1 was taken as control and was treated with vehicle base, while groups 2, 3, and 4 were treated with test formulations; DFS gel marketed emulgel formulation of DFS and DFS-NEG formulation, respectively. In different treated groups, $300 \mathrm{mg}$ of respective formulations was applied on the dorsal surface of the right hind paw with gentle rubbing. After 30 minutes, $20 \mu \mathrm{l}$ of $5 \%$ formalin was subcutaneously injected into the plantar aponeurosis of the right hind paw. The time (in seconds) spent in licking and biting responses of the injected paw was considered as an indicator of pain response. All the measurements were done for $5 \mathrm{~min}$ after formalin injection.

2.7.3. Anti-Inflammatory Activity. Anti-inflammatory activity was evaluated, using the carrageenan-induced rat paw edema method [29]. Animals were divided into four groups, and initial paw volume of all the animals was measured, using plethysmometer. In different treated groups, $300 \mathrm{mg}$ of respective formulations was applied on the dorsal surface of the left hind paw with 50 times gentle rubbing to the treatment groups. Group 1 served as control and was treated with vehicle base, while groups 2, 3, and 4 were treated with test formulations; DFS gel marketed emulgel formulation of DFS and DFS-NEG formulation, respectively. Edema was induced for $30 \mathrm{~min}$. After administration of test and standard drugs by subplantar injection of freshly prepared $1 \%$ carrageenan solution $(100 \mu \mathrm{L})$ in distilled water to the left hind paws of animals of all the groups, change in paw volume was calculated as an initial response, early-phase response $(1 \mathrm{~h})$, and late-phase response $(4 \mathrm{~h})$. The $\%$ inhibition of paw edema was estimated by comparing paw volume of treated groups with that of mean paw volume of control group (29).

2.8. Statistical Analysis. The values were expressed as mean \pm SD. The data were analyzed using one-way ANOVA, followed by post hoc Tukey-Kramer tests using PASWs18
(IBMSPSS software, San Diego, CA, USA). The values of $p<0.05$ were considered significant.

\section{Results and Discussion}

3.1. Screening of Formulation Ingredients. All ingredients chosen for the formulation development were pharmaceutically acceptable. The surfactant, along with cosurfactant used in this formulation, is expected to increase permeation across the skin. Cosurfactant also helps in improving the degree of nanoemulsification [20]. The drug should be completely soluble in the selected oil phase to ensure the stability of nanoemulsion. Due to its higher concentration gradient, it will help in increasing the therapeutic efficacy by boosting the percutaneous drug permeation. The solubility of DFS was found to be exceptionally high in clove oil $(>500 \mathrm{mg} / \mathrm{g})$. Cloves in the form of oil can be used as an antiinflammatory lotion as well. It is used as a topical application to relieve pain and inflammation in traditional system of medicine since ancient time. Recently, Grespan et al. has reported the efficacy of eugenol (a compound obtained from clove oil) to ameliorate experimental arthritis induced by collagen in murine models and suggested it as a useful supplement in treatment of human arthritis [30]. However, after considering the required hydrophilic-lipophilic balance (HLB) of oil phase to develop into NE system, clove oil along with isopropyl myristate in a ratio of $2: 1$ was selected. Since the addition of volatile oils has already proven to increase the topical permeability, further volatile oil (eucalyptus oil and peppermint oil in $1: 1$ ) was also incorporated into the chosen oil system. Finally, clove oil and isopropyl myristate together with volatile oil (2:1:1 ratio) was selected as an optimized oil phase for the development of topical NE of DFS.

The selected surfactant should be capable of decreasing the interfacial tension to a minimum level to help the process of fine dispersion, by offering a flexible film which can easily wrap over the droplets. Also the selected surfactant must be with the proper lipophilic nature to give the optimum curvature at interface. Among the different nonionic surfactants like Tween 20, Tween 80, Cremophor EL, and Cremophor RH, Tween 20 exhibited a highest emulsification property for the used oil phase. However, it is not always achievable to accomplish the desired interfacial area by using one surfactant. Therefore, one more surfactant is introduced to the system, to improve the emulsification efficiency and to obtain a small droplet size. It also aids in improving skin permeation as well as decreases the possibility of skin irritation due to the high concentration of surfactant. The combined property of the two surfactants may be additive but should be noted that the absorption of one surfactant is not negatively interfering the absorption of the second and also the formation of mixed micelle is not reducing the concentration of amphiphilic molecules which are freely available. The second amphiphile is known as cosurfactant [22]. The cosurfactant ultimately increases the stability performance of the NE. Cosurfactants were selected based on the NE region attained in phase behavior study. Nanoemulsion area in the phase diagram represents the possibility of emulsification effectiveness of the selected 
surfactant system. A big nanoemulsion area in phase diagram shows or represents a greater nanoemulsification efficiency of the chosen cosurfactant system [20]. Among the various cosurfactants including PEG 400, ethanol, and propylene glycol, PEG 400 exhibited a higher emulsification ability for the chosen oil and surfactant combination evidenced by phase diagram study. Even though ethanol also showed a good efficacy, it was not considered in the design of $\mathrm{NE}$ formulation due to regulatory restrictions.

3.2. Phase Behavior Study. Phase behavior of DFS-loaded NE was performed by building pseudoternary phase diagram by the use of the aqueous titration technique. In the ternary phase diagram, large liquid crystals (LCs) region and a relatively smaller nanoemulsification area were observed. The phase diagram of ternary system consists of LC region near surfactant-rich apex, while the NE region is observed towards aqueous-rich apex. Furthermore, the addition of PEG 400 along with Tween 20 changed the LC area in the phase diagram to easily flowable nanoemulsion region. It is probably due to increased penetration of the oil phase into the hydrophobic area of the monomers of surfactant [22]. Moreover, Smix ratio $(\mathrm{Km})$ has also significant effect on the phase behavior of DFS-loaded NE system. It was found that raise in the concentration of cosurfactant as compared to surfactant (like $\mathrm{Km} 1: 2,1: 3$, and 1:4) resulted in decreasing the area of nanoemulsifying region, but the spontaneity of nanoemulsification improved greatly. The increase in the concentration of surfactant compared to cosurfactant (like $\mathrm{Km} 2$ : 1) resulted in an increase in the area of nanoemulsifying region due to the significant improvement in solubilization of oil concentration used in nanoemulsion formation. However, a further increase in $\mathrm{Km}$ (like $3: 1$ and $4: 1$ ) resulted in a gradual decrease in the area of nanoemulsifying region with the appearance of LC region. The phase behavior study indicated that Tween 20 along with PEG 400 at $K m$ 2:1 found to be optimum for the development of DFS-loaded NE in presence of oil phase consists of clove oil along with isopropyl myristate in a ratio of $2: 1$ (Figure 1).

3.3. Preparation of DFS-Loaded NE. The selected formulation components and optimized \% composition of components based on the ability of pseudoternary system comprising oil, surfactant, and cosurfactant as Smix and aqueous phase to form stable NE were vortexed for few minutes to obtain a clear, transparent, stable isotropic system. It was prepared by dissolving the DFS in the optimized oil phase (clove oil and isopropyl myristate along with volatile oil in a ratio of $2: 1: 1$ ) by vortex mixing for few minutes. After that, optimized Smix that is Tween 20 as well as PEG 400 (2:1 ratio) and water phase were added instantaneously with vortex mixing. The percentage composition of final formulation of DFS-loaded NE is shown in Table 1.

3.4. Characterization of DFS-Loaded NE. The term thermodynamic stability means a system in its lowest energy level or else in chemical equilibrium with its environment

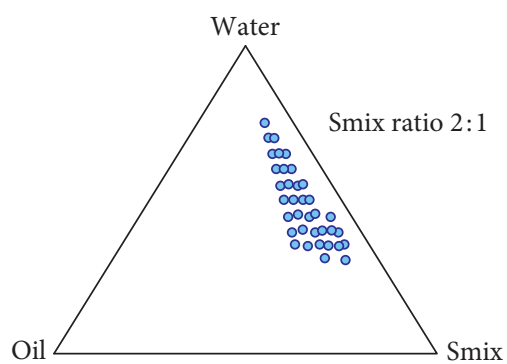

Figure 1: Pseudoternary phase diagram of DFS-NE at $K m$ 2:1.

TABle 1: Percentage composition of optimized DFS-NE formulation.

\begin{tabular}{lcc}
\hline S. no. & Ingredients & \% composition $(\mathrm{w} / \mathrm{w})$ \\
\hline 1 & Diclofenac sodium & 1.0 \\
2 & Clove oil & 10.0 \\
3 & Isopropyl myristate & 5.0 \\
4 & Tween 20 & 31.35 \\
5 & PEG 400 & 14.65 \\
6 & Peppermint oil & 2.50 \\
7 & Eucalyptus oil & 2.50 \\
8 & Distilled water & q.s. to 100 \\
\hline
\end{tabular}

[22]. The thermodynamic tests will help to get out of the presence of metastable formulations. DFS-loaded NE optimized from phase diagram evaluation was selected for accelerated stability tests including heating-cooling as well as freeze-thaw cycles as well as centrifugation. It was found that optimized formulation system did not show any sign of precipitation, phase separation, and creaming. No coalescence and cracking was found during stress stability study. Average droplet size of optimized DFS-loaded NE was found to be $64.07 \pm 2.65 \mathrm{~nm}$ with polydispersity index (PDI) $0.238 \pm 0.02$ (Figure 2). Results confirmed the uniformity in size distribution of droplets. Furthermore, the magnitude of the surface charge will also play a major role in emulsion stability. It is observed the coalescence is prevented when high electrical repulsive forces appear between NE droplets. On the other hand, a decrease in the force of electrostatic repulsion causes phase separation [20]. Zeta potential of the optimized DFS-loaded NE was determined (Figure 3). The $-39.06 \pm 1.23 \mathrm{mV}$ value of zeta potential of DFS-loaded NE showed that the optimized formulation was negatively charged. Negative charge on the optimized DFS-loaded NE may be due to the occurrence of the anionic group which are present in the fatty acids and glycols present in the oil, surfactant, or cosurfactant. The droplet size and morphology optimized DFS-NEG were found to be spherical and less than $100 \mathrm{~nm}$ under polarized and TEM microscopy (Figure 4). The content of DFS in the optimized formulation was estimated by RP-HPLC analysis with UV detector at $254 \mathrm{~nm}$. The percentage content of DFS in the optimized formulation was found to be $99.86 \pm 0.43 \%$.

3.5. Formulation of DFS Containing NEG. Carbopol 980 is crosslinked polyacrylic acid and widely used $\mathrm{pH}$-sensitive polymer to prepare gel formulation for topical application. 


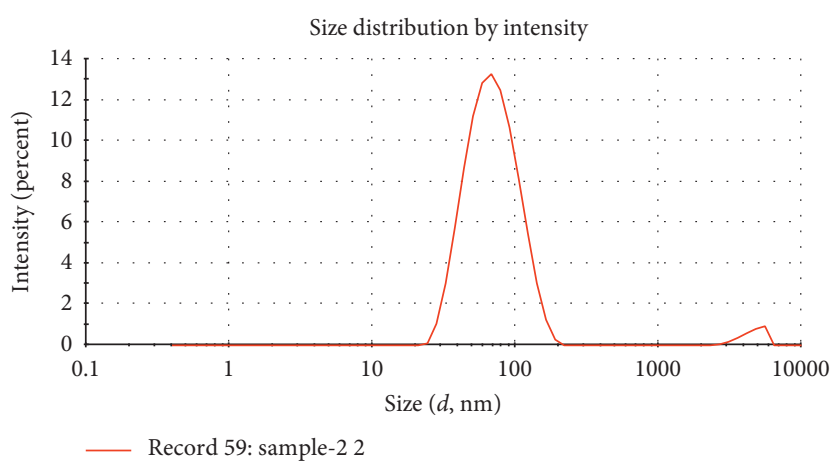

Figure 2: Droplet size distribution of DFS-NE (Z-average $=64.07 \mathrm{~nm}$ with $\mathrm{PdI}=0.238$ ).

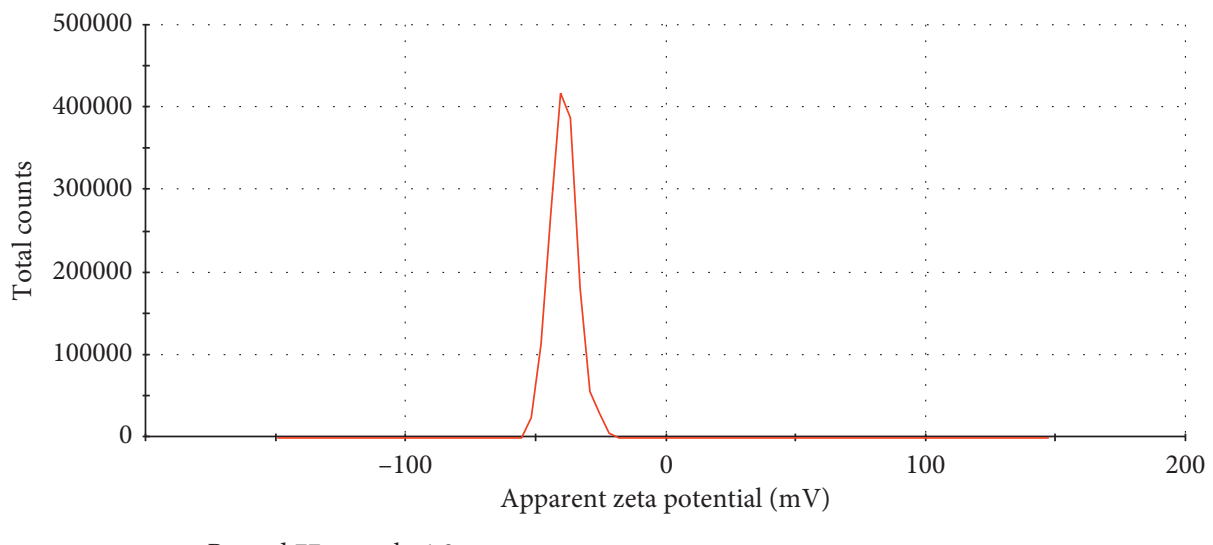

— Record 77: sample-1 2

FIgURE 3: Zeta potential distribution of DFS-NE $(\zeta=-39.06 \mathrm{mV})$.

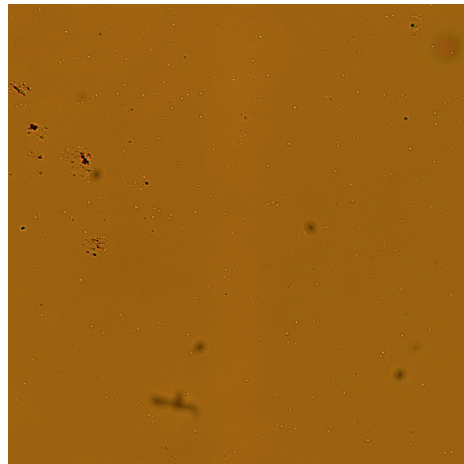

(a)

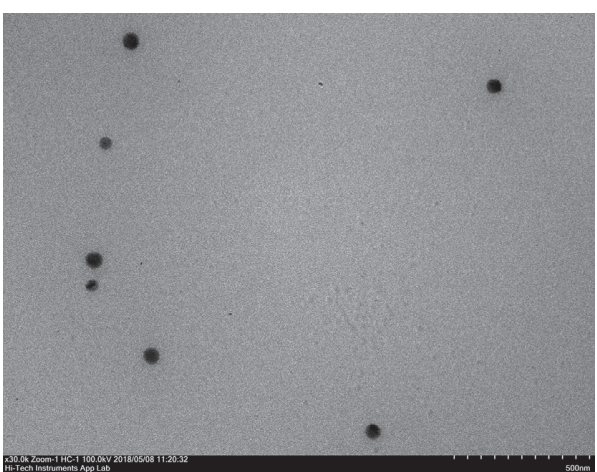

(b)

FIGURE 4: Droplet size and morphology of DFS-NE under polarized and TEM microscopy.

Triethanolamine is used to neutralize the carboxylic groups of the polymers so that aqueous dispersion of Carbopol 980 converted into gel state near to skin $\mathrm{pH}$ [31]. For topical application, DFS containing NEG was formulated with $0.5 \%$ Carbopol 980 as a gelling agent. $0.5 \%$ w/v of Carbopol 980 was dispersed in an adequate amount of purified water. After uniform dispersion, it was kept for $24 \mathrm{hrs}$ to ensure complete swelling. Then, $5.0 \%$ of glycerin was added to this gel followed by few drops of triethanolamine that resulted in the formation of a gel matrix [24]. The optimized DFS-loaded nanoemulsion was then mixed with the gel matrix $(1: 1$ ratio) with mild stirring to obtain DFS-NEG $(1.0 \mathrm{w} / \mathrm{w})$. The composition of DFS-loaded NEG preparation is presented in Table 2.

3.6. Characterization of DFS Containing NEG. To ensure the safe topical application and compatibility with skin, $\mathrm{pH}$ of the developed DFS-NEG formulation was examined. The $\mathrm{pH}$ of the DFS-NEG was found to be $5.47 \pm 0.12$ that was almost 
TABle 2: Percentage composition of optimized DFS-NEG.

\begin{tabular}{lcc}
\hline S. no. & Ingredients & \% composition (w/w) \\
\hline 1 & Diclofenac sodium & 1.0 \\
2 & Clove oil & 10.0 \\
3 & Isopropyl myristate & 5.0 \\
4 & Tween 20 & 31.35 \\
5 & PEG 400 & 14.65 \\
6 & Peppermint oil & 2.50 \\
7 & Eucalyptus oil & 2.50 \\
8 & Glycerin & 5.0 \\
9 & Carbopol 980 & 0.50 \\
10 & Triethanolamine (TEA) & QS \\
11 & Distilled water & QS to 100 \\
\hline
\end{tabular}

equivalent to the skin acid mantle $\mathrm{pH}$. The viscosity of DFSNEG formulation was found to be $1.10 \pm 0.05 \mathrm{~Pa}$.s at shear stress $50 \mathrm{~Pa}$ and shear rate $45 \mathrm{~s}^{-1}$. The results of rheological studies are shown in Figure 5. Figure 5 shows the rheological profile of placebo gel, and NEG is comparable in behavior, and dispersion of NEG in Carbopol 980 was not affecting its rheology. The developed nanoemulgel illustrates pseudoplastic nature with thixotropic character and was found to be effective carrier system for the delivery of DFS topically. Pharmaceutical formulations in a form of gel with pseudoplastic property are thick or viscous under static conditions and become thinner or less viscous after application of shear-stressed resulting in easy spreadability and improved drug permeation after topical application [32]. The spreading test was applied to facilitate quality control of DFS-NEG since spreading is an important characteristic for preparations designed for skin application. The spreading test revealed that a raise in applied force as weight increases the area of spreading of prepared DFS-NEG. Results confirmed that the optimized formulation has satisfactory spreadability characteristics and is appropriate for topical application. The extrusion behavior of a formulation from the container is an important feature for the duration of its application and also patient acceptance. The outcome of the extrudability test also proved the suitability of the developed system for topical application. The results of drug content uniformity study confirmed that optimized DFS-NEG formulation is perfect in terms of content uniformity as it showed a $99.97 \pm 0.11 \%$ uniformity of drug content.

3.7. In Vitro Drug Release Study. Prior to in vivo study, a preliminary in vitro drug release study was carried out using Franz-diffusion cell using PVDF membrane $(0.22 \mu \mathrm{m})$ on optimized DFS-NEG, marketed emulgel of DFS, and DFS gel (DFS dissolved in PG first and then uniformly dispersed into placebo gel of Carbopol $980(0.5 \% \mathrm{w} / \mathrm{v})$ to finally obtain $1.0 \%$ DFS-gel). The release profile of optimized DFS-NEG, marketed emulgel of DFS, and DFS gel was observed for $8 \mathrm{~h}$. The cumulative amount of DFS release $\left(\mathrm{Q}, \mu \mathrm{g} / \mathrm{cm}^{2}\right)$ was plotted against time (h). The cumulative amount of DFS release from developed NEG formulation was $1745.92 \pm 36.45 \mu \mathrm{g} / \mathrm{cm}^{2}$, while the release of DFS from marketed emulgel and conventional gel was found to be
$926.82 \pm 24.49$ and $190.31 \pm 3.94 \mu \mathrm{g} / \mathrm{cm}^{2}$, respectively. In this investigation, concentration of $0.5 \% \mathrm{w} / \mathrm{v}$ Carbopol 980 as a gelling agent produced hydrogel system with desirable viscosity for topical application and optimum rate of DFS release from gel matrix. Zheng et al. reported that incorporating nanoemulsion in a hydrogel system of different concentrations of gelling agent to produce nanoemulgel influences the release of drug from the gel matrix [33]. The increase in the concentration of the gelling agent in the gel matrix decreases the rate of release, and vice versa. This results showed that the drug release profile of DFS from NEG is significantly $(p<0.05)$ higher as compared to marketed emulgel and conventional gel formulation (Figure 6). The results of current investigation were found to be consistent with previous finding, demonstrating that NSAIDs encapsulated into o/w emulsions of submicron/ nanodimension had enhanced transdermal transport behavior (10).

The drug release profile was applied to various mathematical models (first-order model, zero-order model, Higuchi model, and Korsmeyer-Peppas model) to determine the release mechanism based on the highest correlation coefficient $\left(r^{2}\right)$ value. The release kinetics of DFS from NEG formulations best fitted into the Higuchi diffusion model $\left(r^{2}=0.9977\right)$ because of highest $r^{2}$ compared to the firstorder model $\left(r^{2}=0.9928\right)$, zero-order model $\left(r^{2}=0.9093\right)$, and Korsmeyer-Peppas model $\left(r^{2}=0.9032\right)$. Therefore, the mechanism for DFS release from developed NEG formulation is diffusion. The results of current investigation were found to be consistent with previous finding demonstrating that release kinetic of drug from NEG system follows the Higuchi diffusion model (34).

3.8. In Vivo Study. Results of the formalin test (paw licking activity) are shown in Figure 7. All the formulations reduced paw licking activity significantly $(p<0.001$ vs control). The reduction in paw licking activity was approximately 31.23 , 51.19 , and $66.81 \%$ with formulations; DFS gel marketed emulgel of DFS and DFS-NEG formulation, respectively, as compared to control group $(p<0.001)$. These results suggested that developed DFS-NEG formulations possessed relatively better analgesic activity compared to marketed emulgel of DFS and DFS gel. 


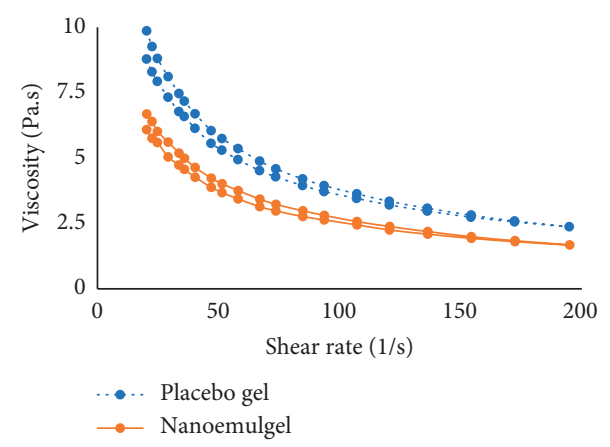

(a)

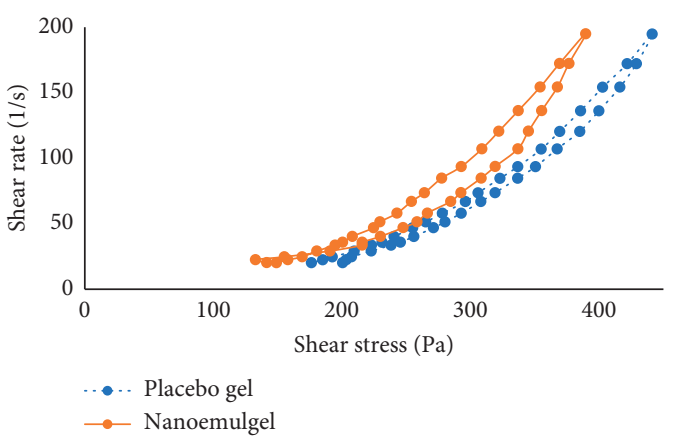

(b)

Figure 5: Rheological profile of 0.5\% (w/v) of Carbopol 980 placebo gel and nanoemulgel: (a) viscosity verses shear rate; (b) shear rate verses shear stress.

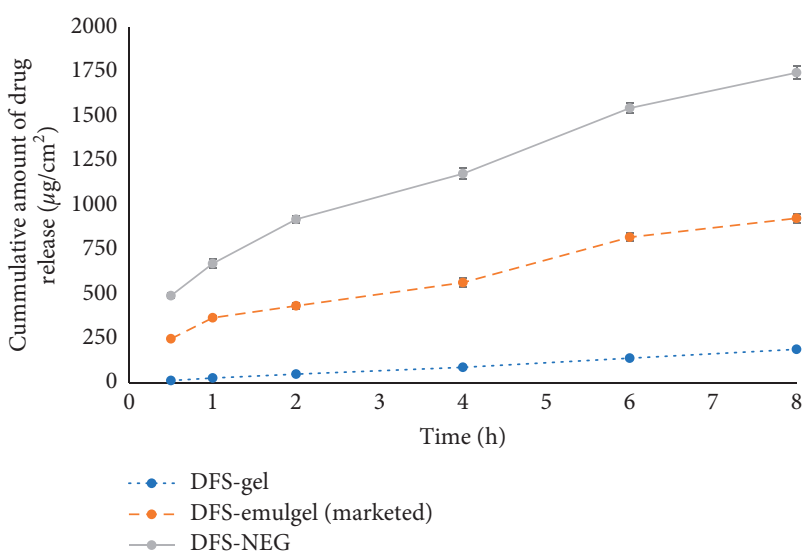

FIgURE 6: In vitro drug release profile of DFS from conventional gel, marketed emulgel, and nanoemulgel.

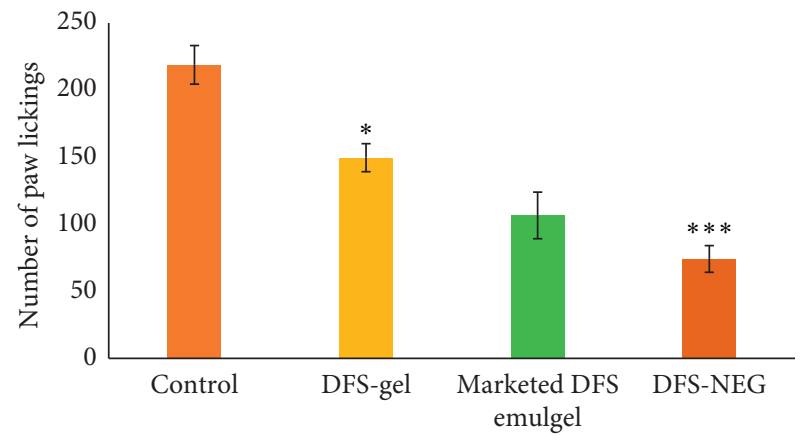

FIGURE 7: Showing the paw licking activity of various formulations. Blank = control; ${ }^{*} p<0.05$ vs blank; ${ }^{* * *} p<0.001$ vs blank.

The outcome of the anti-inflammatory study is presented in Table 3. After $1 \mathrm{~h}$ of induction of paw edema, early-phase response was measured. The results showed that early-phase paw edema was reduced by $41.96 \%$ by marketed emulgel of DFS. The reduction of paw edema was $32.52 \%$ with developed DFS-NEG formulation and $24.91 \%$ with DFS-gel formulation, respectively $[29,30]$. The late-phase response
TABLE 3: Anti-inflammatory activity of test formulations as a measure of percentage (\%) inhibition of paw edema (mean \pm SD).

\begin{tabular}{lccc}
\hline \multirow{2}{*}{ S. no. } & \multirow{2}{*}{ Formulation } & \multicolumn{2}{c}{ \% inhibition of paw edema } \\
& & Early phase & Late phase \\
\hline 1 & DFS gel & $24.91 \pm 1.13^{\mathrm{c}}$ & $44.12 \pm 3.06^{\mathrm{c}}$ \\
2 & Marketed emulgel of DFS & $32.5 \pm 2.45^{\mathrm{b}}$ & $65.89 \pm 3.91^{\mathrm{b}}$ \\
3 & DFS-NEG & $41.96 \pm 2.89^{\mathrm{a}}$ & $78.96 \pm 4.11^{\mathrm{a}}$ \\
\hline a vs b, $p<0.05 ;$ a vs $c, p<0.001$. & &
\end{tabular}

for the percentage inhibition of paw edema was $54.12 \%$, $65.89 \%$, and $78.96 \%$ for DFS-gel, marketed emulgel of DFS, and DFS-NEG formulation, respectively.

\section{Conclusion}

Carbopol 980-based nanoemulgel of DFS was developed, optimized, characterized, and evaluated in animal model. The best nanoemulsion formulation consisted of combination of clove oil, isopropyl myristate together with eucalyptus and peppermint oil as an optimized oil phase, and Tween 20 and PEG 400 as a surfactant and cosurfactant. The zeta potential, droplet size, and PDI of developed nanoemulsions were found to be satisfactory. The $\mathrm{pH}$, viscosity, spreadability, extrudability, and drug content of developed nanoemulgel were found to be satisfactory and suitable for topical application. The rheology behavior of nanoemulgel system was found to be pseudoplastic with the thixotropic property. Moreover, the in vitro release rate, analgesic, and anti-inflammatory activity of developed nanoemulgel of DFS was found to be improved as compared to conventional and marketed gel of DFS. In conclusion, the developed nanoemulgel formulation of diclofenac can be used as a promising carrier for the management of pain and inflammation.

\section{Data Availability}

The data used to support the findings of this study are included within the article. 


\section{Conflicts of Interest}

The authors declare that there are no conflicts of interest.

\section{Acknowledgments}

This project was funded by the Deanship of Scientific Research (DSR) at King Abdulaziz University, Jeddah, under grant no. RG: 5-166-40. The authors, therefore, acknowledge with thanks DSR technical and financial support.

\section{References}

[1] A. V. Hecken, J. I. Schwartz, and M. Depré, "Comparative inhibitory activity of rofecoxib, meloxicam, diclofenac, ibuprofen, and naproxen on COX-2 versus COX-1 in healthy volunteers," The Journal of Clinical Pharmacology, vol. 40, no. 10, pp. 1109-1120, 2000.

[2] E. Ricciotti and G. A. FitzGerald, "Prostaglandins and inflammation," Arteriosclerosis, Thrombosis, and Vascular Biology, vol. 31, no. 5, pp. 986-1000, 2011.

[3] J. P. Peesa, P. R. Yalavarthi, A. Rasheed, and V. B. R. Mandava, "A perspective review on role of novel NSAID prodrugs in the management of acute inflammation," Journal of Acute Disease, vol. 5, no. 5, pp. 364-381, 2016.

[4] K. Nakata, T. Hanai, Y. Take et al., "Disease-modifying effects of COX-2 selective inhibitors and non-selective NSAIDs in osteoarthritis: a systematic review," Osteoarthritis and Cartilage, vol. 26, no. 10, pp. 1263-1273, 2018.

[5] J. M. Janjic, K. Vasudeva, M. Saleem et al., "Low-dose NSAIDs reduce pain via macrophage targeted nanoemulsion delivery to neuroinflammation of the sciatic nerve in rat," Journal of Neuroimmunology, vol. 318, pp. 72-79, 2018.

[6] M. Yang, X. Feng, J. Ding, F. Chang, and X. Chen, "Nanotherapeutics relieve rheumatoid arthritis," Journal of Controlled Release, vol. 252, pp. 108-124, 2017.

[7] M. R. Prausnitz and R. Langer, "Transdermal drug delivery," Nature Biotechnology, vol. 26, no. 11, pp. 1261-1268, 2008.

[8] M. K. Jeengar, S. V. K. Rompicharla, S. Shrivastava et al., "Emu oil based nano-emulgel for topical delivery of curcumin," International Journal of Pharmaceutics, vol. 506, no. 1-2, pp. 222-236, 2016.

[9] N. U. Okur, V. Yozgatlı, M. E. Okur, A. Yoltaş, and P. I. Siafaka, "Improving therapeutic efficacy of voriconazole against fungal keratitis: thermo-sensitive in situ gels as ophthalmic drug carriers," Journal of Drug Delivery Science and Technology, vol. 49, pp. 323-333, 2019.

[10] N. U. Okur, S. Apaydın, N. Ülkü Karabay Yavaşoğlu, A. Yavaşoğlu, and H. Y. Karasulu, "Evaluation of skin permeation and antiinflammatory and analgesic effects ofnew naproxen microemulsion formulations," International Journal of Pharmaceutics, vol. 416, pp. 136-144, 2011.

[11] E. Acioglu, O. Yigit, F. Onur, A. Atas, E. A. Server, and E. Kara, "Ototoxicity associated with topical administration of diclofenac sodium as an otic drop: an experimental animal study," International Journal of Pediatric Otorhinolaryngology, vol. 98, pp. 110-115, 2017.

[12] L. Binder, J. Jatschka, E. M. Kulovits, S. Seeböck, H. Kählig, and C. Valenta, "Simultaneous penetration monitoring of oil component and active drug from fluorinated nanoemulsions," International Journal of Pharmaceutics, vol. 552, no. 1-2, pp. 312-318, 2018.

[13] T. Ilić, S. Savić, B. Batinić et al., "Combined use of biocompatible nanoemulsions and solid microneedles to improve transport of a model NSAID across the skin: in vitro and in vivo studies," European Journal of Pharmaceutical Sciences, vol. 125, pp. 110-119, 2018.

[14] P. Kandadi, M. A. Syed, S. Goparaboina, and K. Veerabrahma, "Albumin coupled lipid nanoemulsions of diclofenac for targeted delivery to inflammation," Nanomedicine: Nanotechnology, Biology and Medicine, vol. 8, no. 7, pp. 1162-1171, 2012.

[15] R. R. Boinpally, S.-L. Zhou, S. Poondru, G. Devraj, and B. R. Jasti, "Lecithin vesicles for topical delivery of diclofenac," European Journal of Pharmaceutics and Biopharmaceutics, vol. 56, no. 3, pp. 389-392, 2003.

[16] L. Tavano, E. Mazzotta, and R. Muzzalupo, "Innovative topical formulations from diclofenac sodium used as surfadrug: the birth of Diclosomes," Colloids and Surfaces B: Biointerfaces, vol. 164, pp. 177-184, 2018.

[17] S. F. Cordery, A. Pensado, W. S. Chiu et al., "Topical bioavailability of diclofenac from locally-acting, dermatological formulations," International Journal of Pharmaceutics, vol. 529, no. 1-2, pp. 55-64, 2017.

[18] J. Ahmad, K. Kohli, S. R. Mir, and S. Amin, "Formulation of self-nanoemulsifying drug delivery system for telmisartan with improved dissolution and oral bioavailability," Journal of Dispersion Science and Technology, vol. 32, no. 7, pp. 958-968, 2011.

[19] S. Kotta, A. W. Khan, S. H. Ansari, R. K. Sharma, and J. Ali, "Formulation of nanoemulsion: a comparison between phase inversion composition method and high-pressure homogenization method," Drug Delivery, vol. 22, no. 4, pp. 455-466, 2015.

[20] J. Ahmad, S. Mir, K. Kohli, and S. Amin, "Quality by design approach for self nanoemulsifying system of paclitaxel," Science of Advanced Materials, vol. 6, no. 8, pp. 1778-1791, 2014.

[21] S. Akhter, M. Anwar, M. A. Siddiqui et al., "Improving the topical ocular pharmacokinetics of an immunosuppressant agent with mucoadhesive nanoemulsions: formulation development, in-vitro and in-vivo studies," Colloids and Surfaces B: Biointerfaces, vol. 148, pp. 19-29, 2016.

[22] J. Ahmad, S. R. Mir, K. Kohli, and S. Amin, "Effect of oil and co-surfactant on the formation of Solutol HS 15 based colloidal drug carrier by Box-Behnken statistical design," Colloids and Surfaces A: Physicochemical and Engineering Aspects, vol. 453, pp. 68-77, 2014.

[23] J. Ahmad, S. R. Mir, K. Kohli et al., "Solid-nanoemulsion preconcentrate for oral delivery of paclitaxel: formulation design, biodistribution, and $\gamma$-scintigraphy imaging," BioMed Research International, vol. 2014, Article ID 984756, 12 pages, 2014.

[24] J. Ahmad, A. Gautam, S. Komath, M. Bano, A. Garg, and K. Jain, "Topical nano-emulgel for skin disorders: formulation approach and characterization," Recent Patents on Antiinfective Drug Discovery, vol. 14, no. 1, pp. 36-48, 2019.

[25] U. Shinde, S. Pokharkar, and S. Modani, "Design and evaluation of microemulsion gel system of nadifloxacin," Indian Journal of Pharmaceutical Sciences, vol. 74, no. 3, pp. 237-247, 2012.

[26] S. A. Al-Suwayeh, E. I. Taha, F. M. Al-Qahtani, M. O. Ahmed, and M. M. Badran, "Evaluation of skin permeation and analgesic activity effects of carbopol lornoxicam topical gels containing penetration enhancer," The Scientific World Journal, vol. 2014, Article ID 127495, 9 pages, 2014.

[27] M. A. Barkat, J. Ahmad, A. R. Harshita et al., "Formulation design of micronized silver sulfadiazine containing aloe vera 
gel for wound healing," Current Bioactive Compounds, vol. 12, no. 2, pp. 63-68, 2016.

[28] B. Hajjar, K.-I. Zier, N. Khalid, S. Azarmi, and R. Löbenberg, "Evaluation of a microemulsion-based gel formulation for topical drug delivery of diclofenac sodium," Journal of Pharmaceutical Investigation, vol. 48, no. 3, pp. 351-362, 2018.

[29] M. A. Khan, S. Kumar, J. Sastry, A. Gupta, and S. Ahmad, "Analgesic and anti-inflammatory potential of three new topical polyherbal formulations in wistar rats," Annals of Phytomedicine, vol. 4, pp. 90-93, 2015.

[30] R. Grespan, M. Paludo, H. D. P. Lemos et al., “Anti-arthritic effect of eugenol on collagen-induced arthritis experimental model," Biological and Pharmaceutical Bulletin, vol. 35, no. 10, pp. 1818-1820, 2012.

[31] M. T. Islam, N. Rodríguez-Hornedo, S. Ciotti, and C. Ackermann, "Rheological characterization of topical carbomer gels neutralized to different $\mathrm{pH}$," Pharmaceutical Research, vol. 21, no. 7, pp. 1192-1199, 2004.

[32] C. H. Lee, V. Moturi, and Y. Lee, "Thixotropic property in pharmaceutical formulations," Journal of Controlled Release, vol. 136, no. 2, pp. 88-98, 2009.

[33] M. A. Morsy, R. G. Abdel-Latif, A. B. Nair et al., "Preparation and evaluation of atorvastatin-loaded nanoemulgel on wound-healing efficacy," Pharmaceutics, vol. 11, no. 11, p. 609, 2019. 